CLINICAL STUDY

\title{
Subcutaneous hydrocortisone administration for emergency use in adrenal insufficiency
}

\author{
Stefanie Hahner*, Stephanie Burger-Stritt* and Bruno Allolio \\ Endocrinology and Diabetes Unit, Department of Medicine I, University Hospital Wuerzburg, Oberdürrbacher Strasse 6, D-97080 Wuerzburg, Germany \\ (Correspondence should be addressed to S Hahner; Email: hahner_s@medizin.uni-wuerzburg.de)
}

*(S Hahner and S Burger-Stritt contributed equally to this work)

\begin{abstract}
Objective: Evaluation of the pharmacokinetics and safety of s.c. hydrocortisone injection for use in adrenal emergency.

Design: Single-center, open-label, sequence-randomized, crossover study in a tertiary care center.

Patients and methods: Twelve patients with chronic Addison's disease. Comparison of hydrocortisone pharmacokinetics after s.c. and i.m. injection (100 mg) and after s.c. administration of sodium chloride $(0.9 \%)$ respectively at three different visits.

Main outcome measure: maximum serum cortisol $\left(C_{\max }\right)$, time to $C_{\max }\left(t_{\max }\right)$, and time to serum

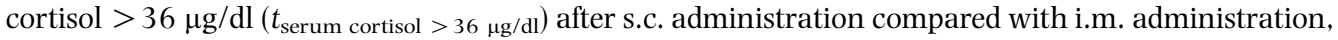
safety, and patient preference.

Results: Serum cortisol increased rapidly and substantially after both i.m. and s.c. injections $\left(C_{\max }\right.$ : $110 \pm 29$ vs $97 \pm 28 \mu \mathrm{g} / \mathrm{dl}, P=0.27, t_{\max }: 66 \pm 51$ vs $91 \pm 34 \mathrm{~min}, P=0.17$, and $t_{\text {serum cortisol }}>$ $36 \mu \mathrm{g} / \mathrm{dl}: 11 \pm 5$ vs $22 \pm 11 \mathrm{~min}, P=0.004$ respectively). Both i.m. and s.c. injections were well tolerated. Eleven $(91.7 \%)$ patients preferred s.c. injection, whereas one patient did not have any preference.

Conclusions: S.c. administration of $100 \mathrm{mg}$ hydrocortisone shows excellent pharmacokinetics for emergency use with only a short delay in cortisol increase compared with i.m. injection. It has a good safety profile and is preferred by patients over i.m. injection.
\end{abstract}

European Journal of Endocrinology 169 147-154

\section{Introduction}

Patients with chronic adrenal insufficiency require lifelong replacement therapy with glucocorticoids. However, even under stable replacement therapy, patients remain at risk of life-threatening adrenal crisis $(1,2)$. An increase in cortisol secretion is an important adaptive mechanism of the organism to deal with stressful events like infectious disease, surgery, and also psychological distress $(3,4,5,6)$. Accordingly, adrenal crisis usually occurs under conditions of a relative cortisol deficiency due to an increased glucocorticoid demand and inadequate replacement. In a retrospective analysis of 444 patients with chronic adrenal insufficiency, we found a frequency of 6.3 adrenal crises/100 patient years (2). Main precipitating factors were gastrointestinal and other infectious diseases. Rapid onset of symptoms was frequently reported (2). Similar observations have been made in a postal survey of Addison's patients from the UK, Canada, Australia, and New Zealand demonstrating that annually $8 \%$ of patients experienced an adrenal crisis with gastrointestinal infection again being the most important trigger (1).
In a prospective analysis of 455 patients, an even higher incidence rate of 7.8 crises/100 patient years was observed (7). Importantly, in this study, in 3\% the outcome of adrenal crisis was fatal. Furthermore, despite established replacement therapy and patient education, mortality of patients with adrenal insufficiency is still increased $(8,9,10)$ and the relative risk to die from infectious disease was 6.6 in a Swedish study (8). Of note, adrenal insufficiency further represented the second most frequent cause of death in a Norwegian analysis (10).

To avoid adrenal crises, patients are educated in adaptation of their glucocorticoid dose under conditions of stress. In addition, they receive an emergency card and should be equipped with an emergency glucocorticoid set. While under conditions of mild-to-moderate stress glucocorticoid dose adaptation can be easily performed by the patients through an increase in the oral replacement dose. In case of vomiting and diarrhea or otherwise severe impairment of the clinical condition, parenteral administration of glucocorticoids by the attending physician is urgently required. As most physicians are not familiar with Addison's disease, i.v. 
glucocorticoid administration is often delayed (11), putting the patient into danger, as serious clinical deterioration may occur within hours.

To enable patients to better deal with imminent adrenal crisis, physicians have started to educate patients and relatives about i.m. hydrocortisone autoinjection. However, different from s.c. auto-injection, self-injection into the muscle often poses a major hurdle to patients and relatives. We, therefore, studied the feasibility and safety of s.c. hydrocortisone administration for emergency use in adrenal insufficiency.

\section{Subjects and methods}

\section{Patients}

Twelve patients with primary adrenal insufficiency currently registered with the outpatient department of the University Hospital Wuerzburg agreed to participate. Inclusion criteria were primary adrenal insufficiency under stable glucocorticoid replacement therapy due to autoimmune adrenalitis or bilateral adrenalectomy (disease duration at least 12 months), age $\geq 18$ years, and ability to comply with the protocol procedures. Exclusion criteria were diabetes mellitus, current infectious disease with fever at the time of investigation, known intolerance to the study drug or constituents of the study drug, oral contraception/ oral estrogens for hormonal replacement therapy, pregnancy or breastfeeding, and renal failure (creatinine $>2.5$ upper limit of normal (ULN)).

The study was approved by the Ethics Committee of the University of Wuerzburg (permit no. 182/11_m) and written informed consent was obtained from all patients before participation. The study was registered at clinicaltrials.gov (clinicaltrials.gov identifier: NCT01450930); in addition, approval was obtained by the federal institute for drugs and medical devices (BfArM). EUDRACT-No.: EudraCTNr. 2011-002687-25.

The primary endpoint was defined as the time needed to reach cortisol levels of $>36 \mu \mathrm{g} / \mathrm{dl}(>1000 \mathrm{nmol} / \mathrm{l})$, as such concentrations may be encountered in severe critical illness $(3,12,13,14)$ and because $36 \mu \mathrm{g} / \mathrm{dl}$ is twice the cutoff level required to pass the short synacthen test representing a maximum ACTH stimulus. The hypothesis was that after s.c. injection, such a serum level can be safely achieved within a time frame of $30 \mathrm{~min}$ with higher patient acceptance compared with i.m. administration. Further end points were key pharmacokinetic data for hydrocortisone: $C_{\max }$, maximal serum concentration; $C_{\text {min }}$, minimal serum concentration; $C_{\mathrm{av}}$, average cortisol plasma concentration; $t_{\max }$, time to $C_{\text {max }}$; AUCt, area under the plasma concentration curve from administration to last observed concentration at time $t$; mean residence time (MRT); and $t_{1 / 2}$, plasma concentration half-life.
Further secondary end points were the safety of s.c. hydrocortisone administration and the response of plasma ACTH to glucocorticoid administration as a measure of glucocorticoid action. Finally, acceptability of the different modes of glucocorticoid administration was assessed by a questionnaire.

\section{Pharmacokinetic studies}

Patients were investigated in the morning (started between 0800 and $1100 \mathrm{~h}$ ). The usual morning dose of hydrocortisone was postponed until the end of the respective visit, whereas fludrocortisone was taken as usual. After assessment of inclusion and exclusion criteria, patients underwent a physical examination. An in-dwelling catheter was placed into a cubital vein. All patients received sodium chloride $0.9 \%$ (divided into $2 \times 1 \mathrm{ml}$ doses) as s.c. injection in the abdominal subcutaneous fat at the first visit and were then randomized concerning the order of the two different hydrocortisone administrations: $100 \mathrm{mg}$ hydrocortisone (Pfizer) in $2 \mathrm{ml}$ solvent subcutaneously (divided in $2 \times 1 \mathrm{ml}$ ) and $100 \mathrm{mg}$ hydrocortisone (Pfizer) in $2 \mathrm{ml}$ solvent intramuscularly in the thigh. Visits were separated by a minimum of 7 days. At every study visit, blood samples were collected at $-10,0,5,10,15$, $20,30,45,60,75,90,120,150,180,210$, and $240 \mathrm{~min}$ after injection. Saliva samples for determination of salivary cortisol were collected at the same time points. In addition, at $-10,0,30,60,90,120,150$, 180,210 , and $240 \mathrm{~min}$, samples for determination of plasma ACTH were collected.

\section{Questionnaires and safety assessment}

Patients received a diary to document any local or systemic adverse events during the 3 days following the intervention. After completion of all three study days, patients further received a questionnaire to collect general information on their adrenal disease and a personal evaluation and rating of the different administration modes. A photographic documentation of the site of injection was performed after injection and at the end of the study visit.

\section{Hormone measurements}

For determination of serum cortisol, an automated luminescence assay was used, which is also used for routine determination of serum cortisol levels (Cortisol Siemens Immulite 2000, Siemens Healthcare Diagnostics, Eschborn, Germany). Intra- and interassay variations are $<10 \%$. Cross-reactivity for aldosterone is $0.1 \%$. Samples with values above the calibration range of $50 \mu \mathrm{g} / \mathrm{dl}$ were reanalyzed after 1:20 dilution with standard solution. 
ACTH levels were also determined with an automated luminescence assay (ACTH Siemens Immulite 2000, Siemens Healthcare Diagnostics). Intra-assay variation was $<10 \%$, and interassay variation was $\leq 10 \%$.

For determination of salivary cortisol levels, a luminescence immunoassay was used (IBL International GmbH, Hamburg, Germany). Intra- and interassay variations were $<5 \%$. Detection range was $0.005-4 \mu \mathrm{g} / \mathrm{dl}$. Levels above the detection range were diluted 1:10 with standard solution.

\section{Pharmacokinetic analysis}

For pharmacokinetic analysis, the maximum $\left(C_{\max }\right)$, minimum $\left(C_{\text {min }}\right)$, and average $\left(C_{\text {av }}\right)$ serum and salivary cortisol concentrations were assessed. In addition, the time until the maximum serum or salivary concentration $\left(t_{\text {max }}\right)$ and the time to a serum concentration of $36 \mu \mathrm{g} / \mathrm{dl}\left(t_{>36 \mu \mathrm{g} / \mathrm{dl}}\right)$ were determined. The area under the concentration-time curve from zero to last sampling time $\left(\mathrm{AUC}_{\mathrm{O}-240}\right)$, MRT, which indicates the average amount of time that a compound spends in a particular system, and elimination half-life $\left(t_{1 / 2}\right)$ were calculated. $C_{\text {max }}, C_{\text {min }}, t_{\text {max }}$, and $t_{>36 \mu \mathrm{g} / \mathrm{dl}}$ were obtained directly from the observed values. $C_{\mathrm{av}}$ was calculated as mean of all values between 5 and $240 \mathrm{~min}$. $\mathrm{AUC}_{0-240}$ was assessed by the trapezoidal rule. MRT was calculated as the area under the moment curve divided by the $\mathrm{AUC}_{0-}$ 240. Elimination half-life was calculated using the formula: $t_{1 / 2}=\ln (2) / \mathrm{ke}$. The elimination rate constant was calculated as follows: $\mathrm{ke}=(\ln (\mathrm{C} 1)-\ln (\mathrm{C} 2)) / \Delta t)$.

\section{Statistical analysis}

Statistical analysis was performed by PASW Statistics 20 (IBM SPSS, IBM Corp.). For comparison of the pharmacokinetics of i.m. vs s.c. administration of $100 \mathrm{mg}$ hydrocortisone, a one-way ANOVA was used. The influence of BMI on pharmacokinetic parameters was examined by calculation of Pearson's correlation coefficient. $\chi^{2}$ test was used for comparison of the patients' preference of administration mode. Data are presented as mean \pm s.D. or median and range. The $95 \%$ CI, minimal (MIN) and maximal (MAX) values, and $P$ values were calculated. Differences were considered as statistically significant when $P<0.05$.

\section{Results}

\section{Study cohort}

The 12 patients (five females and seven males) completed all study visits. Ten patients had been additionally diagnosed with autoimmune thyroiditis and two females suffered from premature ovarian failure. Median age was 47.5 (29-62) years, and median duration of Addison's disease was 11 (1-42) years. BMI was 26.3 (20.8-29.8). All patients were on stable adrenal replacement therapy with hydrocortisone 22.5 (range 15-39) $\mathrm{mg}$ and fludrocortisone 0.09 (0.05-0.15) mg. Eight patients further received levothyroxine replacement due to autoimmune thyroiditis (125 $\mu \mathrm{g} ; 50-175)$. No patient received any medication known to induce cortisol-metabolizing hepatic cytochrome P450 3A4.

\section{Pharmacokinetics of hydrocortisone after s.c. and i.m. administration}

Cortisol levels were undetectable or remained continuously below the normal range after administration of sodium chloride. Pharmacokinetic parameters are given in Tables 1 and 2. Maximum serum cortisol levels after administration of $100 \mathrm{mg}$ hydrocortisone did not differ significantly after i.m. vs s.c. injection $(110 \pm 29$ and $97 \pm 28 \mu \mathrm{g} / \mathrm{dl}$ respectively). Maximum serum cortisol concentrations were reached after $66 \pm 51$ and $91 \pm$ 34 min respectively $(P=0.17)$. No statistical difference was observed between the two administration routes regarding $C_{\mathrm{av}}, \mathrm{AUC}_{0-240}$, MRT, and half-life. However, time until reaching serum cortisol levels above $36 \mu \mathrm{g} / \mathrm{dl}$ significantly differed $(11 \pm 5 \mathrm{~min}$ after i.m. administration vs $22 \pm 11 \mathrm{~min}$ after s.c. administration, $P=0.004)$. Salivary cortisol levels also increased after both i.m. and s.c. administrations of hydrocortisone. Maximum levels did not show statistical difference $(59 \pm 26 \mu \mathrm{g} / \mathrm{dl}$ after i.m., $41 \pm 17 \mu \mathrm{g} / \mathrm{dl}$ after s.c. administration, $P=0.065) . C_{a v}$ was significantly higher after i.m. hydrocortisone administration $(28 \pm 11$ vs $20 \pm 9 \mu \mathrm{g} / \mathrm{dl}, P=0.046)$. Furthermore, $t_{\max }$ was shorter after i.m. hydrocortisone administration $(51 \pm 28$ vs $74 \pm 22 \mathrm{~min}, P=0.036)$. MRT was significantly longer after s.c. hydrocortisone administration $(110 \pm 12$ vs $91 \pm 18$ min after i.m. administration, $P=0.006)$. Halflife and $\mathrm{AUC}_{\mathrm{O}-240}$ did not differ significantly. ACTH levels continuously decreased from $714 \pm 420$ at baseline to $15 \pm 7 \mathrm{ng} / \mathrm{l}$ after i.m. hydrocortisone administration and $984 \pm 712$ at baseline to $18 \pm 7 \mathrm{ng} / \mathrm{l} 240 \mathrm{~min}$ after s.c. hydrocortisone administration with no differences between the two modes of hydrocortisone administration (Fig. 1C).

A significant correlation was observed between BMI and $C_{\max }(r=-0.728, P=0.007), C_{\mathrm{av}}(r=-0.745$, $P=0.005)$, AUC $(r=-0.637, P=0.026)$, and MRT $(r=0.776, P=0.003)$ after s.c. administration of hydrocortisone. No significant correlation with BMI was found for $t_{\max }(r=0.492, P=0.104)$. No correlation between BMI and pharmacokinetic data was seen after i.m. hydrocortisone administration. Patients were further divided into a group with BMI $>25 \mathrm{~kg} / \mathrm{m}^{2}$ $(n=7)$ and patients with a BMI $\leq 25 \mathrm{~kg} / \mathrm{m}^{2}(n=5)$. Comparing pharmacokinetic parameters of s.c. and i.m. injection in the patients with BMI $\leq 25 \mathrm{~kg} / \mathrm{m}^{2}$, no significant differences were observed. In contrast, in the group with BMI $>25 \mathrm{~kg} / \mathrm{m}^{2}$, significant differences 
Table 1 Pharmacokinetic parameters after s.c. and i.m. administration of hydrocortisone (serum cortisol concentrations).

\begin{tabular}{|c|c|c|c|c|c|}
\hline & Mean \pm s.D. & $95 \% \mathrm{Cl}$ & MIN & MAX & $P$ \\
\hline \multicolumn{6}{|c|}{$C_{\max }(\mu \mathrm{g} / \mathrm{dl})$} \\
\hline I.m. & $109.9 \pm 29.2$ & $91.4-128.5$ & 67.3 & 177.0 & \multirow[t]{2}{*}{0.270} \\
\hline S.c. & $96.8 \pm 27.5$ & $79.34-114.3$ & 50.9 & 153.0 & \\
\hline \multicolumn{6}{|c|}{$C_{\mathrm{av}}(\mu \mathrm{g} / \mathrm{dl})$} \\
\hline I.m. & $74.8 \pm 20.0$ & $62.1-87.5$ & 48.8 & 117.1 & \multirow[t]{2}{*}{0.078} \\
\hline S.c. & $60.8 \pm 17.1$ & $79.3-71.7$ & 34.4 & 101.9 & \\
\hline \multicolumn{6}{|c|}{$t_{\max }(\min )$} \\
\hline I.m. & $65.8 \pm 51.3$ & 33.3-98.4 & 20 & 180 & \multirow[t]{2}{*}{0.165} \\
\hline S.c. & $91.3 \pm 33.5$ & $70.0-112.5$ & 45 & 150 & \\
\hline \multicolumn{6}{|c|}{$t_{>36 \mu \mathrm{g} / \mathrm{dl}}(\mathrm{min})$} \\
\hline I.m. & $10.8 \pm 5.2$ & $7.6-14.1$ & 5 & 20 & \multirow{3}{*}{0.004} \\
\hline S.c. & $22.1 \pm 11.2$ & $15.0-29.2$ & 5 & 45 & \\
\hline \multicolumn{5}{|c|}{$\mathrm{AUC}_{0-240}(\mu \mathrm{g} / \mathrm{dl}$ per min$)$} & \\
\hline I.m. & $17997.3 \pm 4332.3$ & $15244.7-20749.9$ & 12789 & 28207 & \multirow[t]{2}{*}{0.318} \\
\hline S.c. & $16335.5 \pm 3608.2$ & $14043.0-18628.1$ & 10208 & 24244 & \\
\hline \multicolumn{6}{|c|}{ MRT (min) } \\
\hline I.m. & $112.1 \pm 10.5$ & $105.5-118.8$ & 100.4 & 130.8 & \multirow[t]{2}{*}{0.116} \\
\hline S.c. & $119.1 \pm 10.2$ & $112.6-125.6$ & 99.7 & 131.1 & \\
\hline \multicolumn{6}{|l|}{$t_{1 / 2}(\mathrm{~h})$} \\
\hline l.m. & $2.2 \pm 1.5$ & $1.3-3.1$ & 1.0 & 6.4 & \multirow[t]{2}{*}{0.900} \\
\hline S.c. & $4.7 \pm 4.7$ & $1.7-7.7$ & 0.8 & 16.6 & \\
\hline
\end{tabular}

$C_{\max }$, maximum concentration; $C_{\mathrm{av}}$, average concentration; $t_{\mathrm{max}}$, time to maximum concentration; $t_{>36} \mu \mathrm{g} / \mathrm{dl}$, time to $>36 \mu \mathrm{g} / \mathrm{dl}$ (for conversion in nmol/l multiply with 27.6); $\mathrm{AUC}_{0-240}$, area under the concentration-time curve from zero to last sampling time; MRT, mean residence time; $t_{1 / 2}$, half-life time; MIN, minimum; MAX, maximum. Analyses were performed by one-way ANOVA. For conversion of cortisol concentration from $\mu \mathrm{g} / \mathrm{dl}$ to $\mathrm{nmol} / \mathrm{l}$ multiply with 27.6 .

between s.c. and i.m. injection were observed for $C_{\mathrm{av}}$ $(53 \pm 9$ vs $73 \pm 18 \mu \mathrm{g} / \mathrm{dl}$ for s.c. and i.m., respectively, $P=0.02), \quad t>36 \mu \mathrm{g} / \mathrm{dl} \quad(27 \pm 11$ vs $10 \pm 5 \mathrm{~min}$, $P=0.002)$, and MRT $(125 \pm 6$ vs $113 \pm 12 \mathrm{~min}$, $P=0.036$; Fig. 2). Similarly, no differences between the BMI groups were observed after i.m. injection, whereas values for $t_{\max }$ and MRT differed significantly between the BMI groups after s.c. injection (Supplementary Tables 1 and 2, see section on supplementary data given at the end of this article).

\section{Patient questionnaires}

Six $(50 \%)$ patients reported that they had already experienced an adrenal crisis since diagnosis of Addison's disease. All patients were equipped with an emergency card and had received instructions regarding dose adaptation of hydrocortisone. In general, the patients rated their coping with the disease as "very good' $(n=3)$ or 'good' $(n=9)$. However, five patients indicated that they felt restricted in their leisure time

Table 2 Pharmacokinetic parameters after s.c. and i.m. administration of hydrocortisone (salivary cortisol concentrations).

\begin{tabular}{|c|c|c|c|c|c|}
\hline & Mean \pm S.D. & $95 \% \mathrm{Cl}$ & MIN & MAX & $P$ \\
\hline \multicolumn{6}{|c|}{$C_{\max }(\mu \mathrm{g} / \mathrm{dl})$} \\
\hline I.m. & $58.7 \pm 26.0$ & $42.2-75.2$ & 24.5 & 111.2 & \multirow[t]{3}{*}{0.065} \\
\hline S.c. & $41.3 \pm 16.9$ & $30.6-52.1$ & 16.3 & 69.3 & \\
\hline \multicolumn{5}{|c|}{$C_{\mathrm{av}}(\mu \mathrm{g} / \mathrm{dl})$} & \\
\hline I.m. & 27. $9 \pm 10.5$ & $21.2-34.5$ & 13.7 & 49.4 & \multirow[t]{2}{*}{0.046} \\
\hline S.c. & 19. $6 \pm 8.5$ & $14.2-25.0$ & 8.1 & 37.0 & \\
\hline \multicolumn{6}{|c|}{$t_{\max }(\min )$} \\
\hline I.m. & $51.3 \pm 27.5$ & $33.8-68.7$ & 30 & 120 & \multirow[t]{2}{*}{0.036} \\
\hline $\begin{array}{l}\text { S.c. } \\
\text { AUC. }\end{array}$ & $73.8 \pm 21.7$ & $60.0-87.5$ & 45 & 120 & \\
\hline I.m. & $6177.9 \pm 2096.5$ & $4845.8-7509.9$ & 3379.2 & 10503.5 & \multirow[t]{2}{*}{0.238} \\
\hline S.c. & $5196.4 \pm 1862.8$ & $4012.8-6380.0$ & 2335.6 & 8329.3 & \\
\hline \multicolumn{6}{|c|}{ MRT (min) } \\
\hline I.m. & $91.3 \pm 17.8$ & $80.0-102.6$ & 65.8 & 128.5 & \multirow[t]{3}{*}{0.006} \\
\hline S.c. & $110.2 \pm 12.3$ & $102.4-118.0$ & 92.0 & 131.3 & \\
\hline \multicolumn{5}{|l|}{$t_{1 / 2}(h)$} & \\
\hline I.m. & $4.6 \pm 9.8$ & $-1.7-10.8$ & 0.4 & 35.3 & \multirow[t]{2}{*}{0.666} \\
\hline S.c. & $3.3 \pm 7.2$ & $1.14-5.4$ & 0.7 & 10.7 & \\
\hline
\end{tabular}

$C_{\max }$, maximum concentration; $C_{\mathrm{av}}$, average concentration; $t_{\mathrm{max}}$, time to maximum concentration; AUC $C_{0-240}$, area under the concentration-time curve from zero to last sampling time; MRT, mean residence time; $t_{1 / 2}$, half-life time; MIN, minimum; MAX, maximum. Analyses were performed by one-way ANOVA. For conversion of cortisol concentration from $\mu \mathrm{g} / \mathrm{dl}$ to $\mathrm{nmol} / \mathrm{l}$ multiply with 27.6 . 

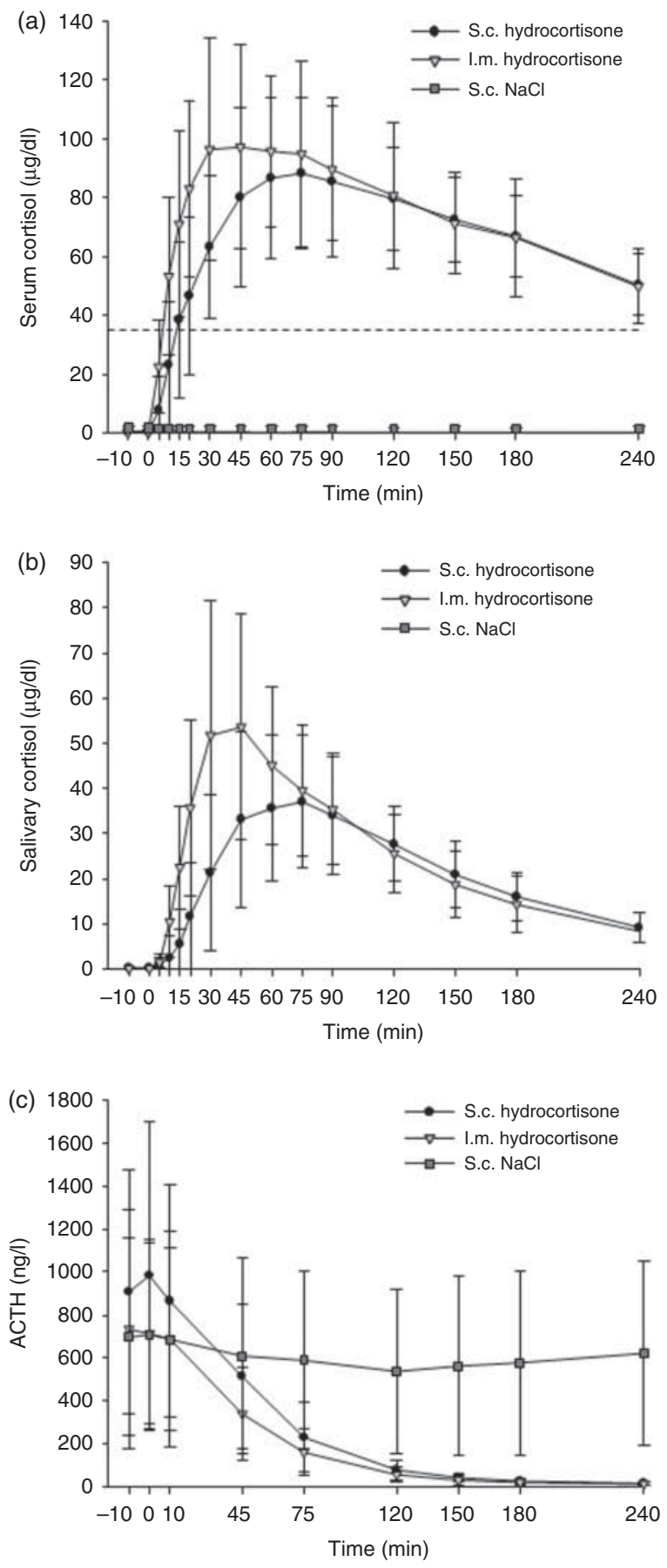

Figure 1 Comparison of s.c. and i.m. administration of hydrocortisone; s.c. administration of sodium chloride was used as control. (a) Curves represent mean serum cortisol concentrations vs time. Dotted line indicates the $36 \mu \mathrm{g} / \mathrm{dl}(1000 \mathrm{~nm} / \mathrm{l})$ threshold. (b) Curves represent mean salivary cortisol concentrations vs time. (c) Curves represent plasma ACTH concentrations vs time.

activities. Eleven of the 12 study participants were of the opinion that being able to self-inject hydrocortisone would make them feel safer. Nine patients indicated that this would also improve their quality of life. Regarding the mode of administration of hydrocortisone, 11 patients preferred s.c. administration $(P<0.01)$, whereas one patient did not have any preference.

\section{Adverse events}

Both the i.m. and s.c. injections were well tolerated. In total, 21 adverse events were documented: after i.m. administration of hydrocortisone, pain in the whole leg of the side of injection $(n=2)$, local pain at the injection site $(n=2)$, burning at the injection site $(n=2)$, and local feeling of pressure at the injection site $(n=7)$; after s.c. administration of hydrocortisone, local pain at the injection site $(n=1)$, itching at the injection site $(n=1)$, and burning at the injection site $(n=2)$; and after s.c. administration of sodium chloride $0.9 \%$, burning at injection site $(n=2)$, local pain at the injection site $(n=1)$, and itching at injection site $(n=1)$. All symptoms were mild, did not require any intervention, and resolved within 15-240 min after onset.

\section{Discussion}

Despite careful patient education, provision of an emergency card, and an emergency steroid selfinjection kit, patients with adrenal insufficiency on stable replacement are at risk of adrenal crisis $(1,2)$ with evidence of increased mortality due to such crises $(7,8,9,10)$. This observation indicates that current prevention strategies need to be improved. Patients are educated to adapt their oral hydrocortisone dose in case of stressful events. In general, high cortisol levels may be obtained after a sufficient dose of oral hydrocortisone. However, in case of incipient adrenal crisis, these levels should be achieved within a very short time frame and enteral absorption of hydrocortisone may be largely impaired, e.g. in case of gastroenteritis. Selfadministration of parenteral hydrocortisone in case of impending crisis holds great potential to reduce crisisassociated morbidity and mortality. Accordingly, in some centers, training of i.m. self-injection of hydrocortisone has become part of patient education $(11,15)$. However, many patients are reluctant to use i.m. selfinjection, while s.c. self-injection seems to be more acceptable to patients. Accordingly, our study provided clear evidence that patients prefer s.c. over i.m. injection.

The major finding of our study is the high efficacy of s.c. hydrocortisone administration to safely reach target serum cortisol concentrations. When planning this study, we hypothesized that the time to reach a cortisol concentration of $36 \mu \mathrm{g} / \mathrm{dl}$ would allow a reasonable evaluation of the clinical utility of s.c. hydrocortisone administration. This assumption is based on the established cutoff levels of the short synacthen test, a maximum ACTH challenge, where a cortisol increase to $18 \mu \mathrm{g} / \mathrm{dl}(500 \mathrm{nmol} / \mathrm{l})$ after $30 \mathrm{~min}$ 

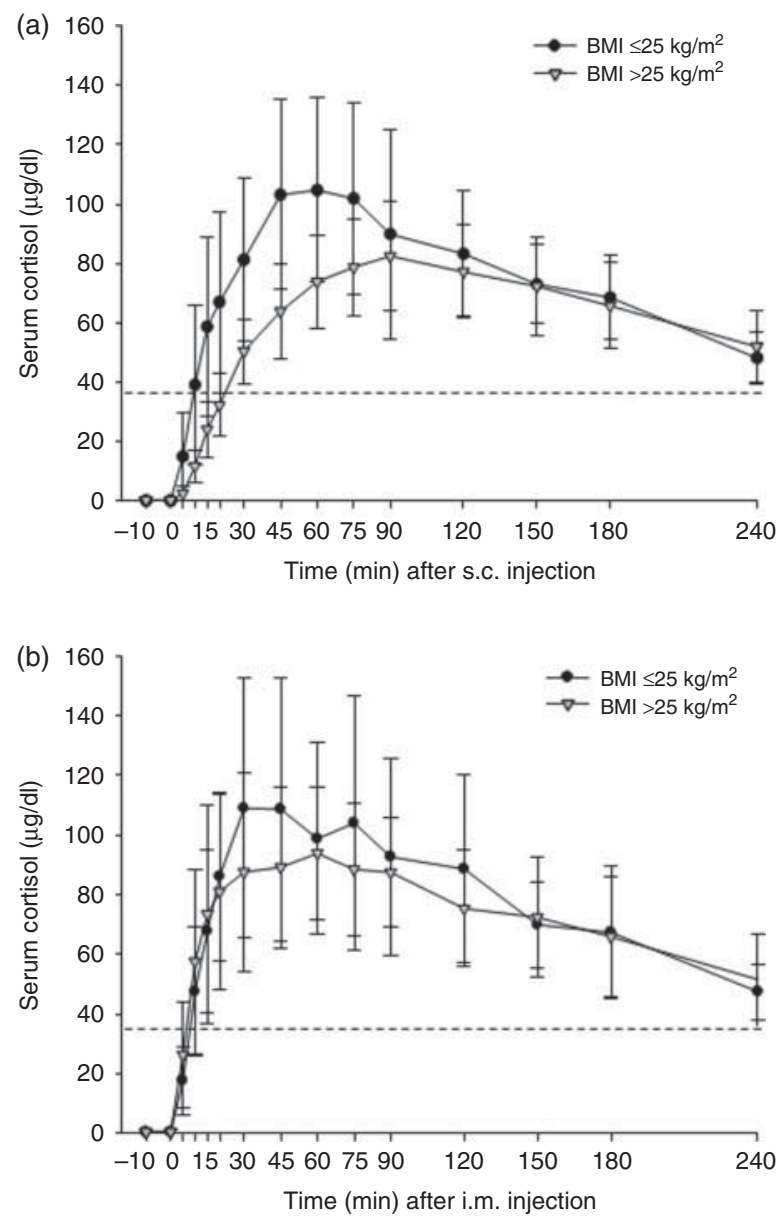

Figure 2 Comparison of patients with a BMI $\leq 25 \mathrm{~kg} / \mathrm{m}^{2}$ vs patients with a BMI $>25 \mathrm{~kg} / \mathrm{m}^{2}$ after s.c. (a) and i.m. (b) administration of hydrocortisone. Dotted line indicates the $36 \mu \mathrm{g} / \mathrm{dl}(1000 \mathrm{~nm} / \mathrm{l})$ threshold.

is considered to show an adequate adrenal response. By doubling this value, a high safety margin concerning sufficient glucocorticoid availability can be assumed. Furthermore, patients in intensive care with such cortisol concentrations are considered sufficiently protected from cortisol deficiency, and in general, the average serum cortisol concentration remains below these concentrations in patients with critical illness $(3,12,13,14,16)$. With a mean time of $22 \mathrm{~min}$ (95\% CI 15-29 $\mathrm{min}$ ) to reach this target concentration, s.c. administration of hydrocortisone, therefore, clearly fulfills the requirements for rapidly covering the increased glucocorticoid needs in case of emergency. However, compared with i.m. hydrocortisone administration, the time to reach this target concentration was significantly delayed by $11 \mathrm{~min}$. The disadvantage of such a delay seems to be minor compared with higher ease and acceptance of s.c. hydrocortisone administration, which may facilitate an earlier self-injection compensating for the short delay in serum cortisol increase.
It may be possible that in case of shock status, s.c. absorption might be diminished. However, the aim of training patients in performing self-injection is rather to prevent shock in incipient adrenal crisis and to shorten the time interval until parenteral glucocorticoids are provided by easy-to-perform and timely self-administration of hydrocortisone than to treat adrenal crisis in a status that needs professional health care with i.v. glucocorticoids. The critical time frame in which we believe that s.c. self-administration of hydrocortisone has its value is incipient adrenal crisis at a time when the patient is still able to treat himself/herself until professional health care (and i.v. hydrocortisone) can be provided. Our aim is not to fully replace i.m. selfinjection, which still has its value. However, patients may now choose their most preferred administration mode. In our study, most patients preferred s.c. injection over i.m. injection, although s.c. injection was given as two single injections compared with one single i.m. injection.

Furthermore, it might be possible that administration of two single doses might have changed the pharmacokinetic profile in comparison with a single administration. As $100 \mathrm{mg}$ hydrocortisone was only available as a $2 \mathrm{ml}$ solution and as the volume of fluid administered subcutaneously as a single bolus should not exceed $1 \mathrm{ml}$, we had to perform two simultaneous injections.

Nevertheless, a good absorption of hydrocortisone after s.c. administration was observed in our study. We, therefore, believe that a potential further delay of the increase in cortisol levels after s.c. administration of a single injection would still by far be outweighed by the advantages of higher patient acceptance and higher probability to be used in case of emergency, which may be life-saving. In fact, the cortisol concentrations after both i.m. and s.c. hydrocortisone administrations clearly reach supraphysiological levels even if compared with the most severe stress in patients with intact adrenal function and remain supraphysiological for several hours, although cortisol concentrations start to decline after $120 \mathrm{~min}$. However, in incipient adrenal crisis, it may be better to provide excess glucocorticoids for a few hours rather than to under-replace the patient. This approach is also a common practice in perioperative glucocorticoid coverage, as current replacement strategies lead to greatly increased cortisol concentrations compared with mean cortisol concentrations in patients without adrenal disorders undergoing surgery. However, unexpected complications like postoperative septic episodes or severe bleeding may require high glucocorticoid availability, which may not be adequately covered with a lower replacement dose and carry the risk that the higher need in an individual patient is not detected in time.

It may be expected that patients would prefer one single injection over two injections. Further developments should, therefore, aim at the development of a hydrocortisone preparation, which is suitable for a 
single s.c. injection. However, in our study, s.c. injection was still preferred over i.m. injection, although two simultaneous injections had to be performed.

The findings for salivary cortisol are similar to those for serum cortisol, indicating a rapid rise in free bioavailable cortisol. Furthermore, suppression of plasma ACTH was not significantly different between i.m. and s.c. hydrocortisone administrations, supporting the view of similar glucocorticoid action due to early ceiling effects after both administrations.

A number of pharmacokinetic parameters differed significantly between i.m. and s.c. administrations, and for other parameters, the small number of patients may have contributed to a lack of significance. This is not unexpected as a slightly faster systemic availability after i.m. administration has been described for a number of drugs, which is mainly due to a higher perfusion of muscle compared with subcutaneous fat (17). A significant correlation was observed between BMI and $C_{\text {max }}(r=-0.728, P=0.007)$ but not between BMI and $t_{\max }$. However, $t_{\max }$ significantly differed between patients with BMI $>25$ and $\leq 25 \mathrm{~kg} / \mathrm{m}^{2}$. In the patients with BMI $\leq 25 \mathrm{~kg} / \mathrm{m}^{2}$, pharmacokinetic parameters after s.c. and i.m. injection were comparable. In contrast, significant differences were observed for $C_{\mathrm{av}}, t>36 \mu \mathrm{g} / \mathrm{dl}$ and MRT in the overweight patients (BMI $>25 \mathrm{~kg} / \mathrm{m}^{2}$ ). Thus, the slightly delayed cortisol increase after s.c. hydrocortisone administration in overweight/obese patients should be taken into account if using s.c. hydrocortisone as a crisis prevention measure. Nevertheless, supraphysiological cortisol levels were rapidly and consistently obtained also in these participants after s.c. administration.

Self-administration of hydrocortisone may not only improve management of adrenal insufficiency but also be associated with significant risks, as the patient may delay or cancel emergency care by a physician wrongly believing that self-management will be sufficient. Thus, it is recommended that self-injection of hydrocortisone should invariably trigger a contact to a physician for further assessment. However, it is our experience and that of others (11) that the time from contacting an emergency physician to the injection of hydrocortisone frequently exceeds several hours, even if the emergency card is shown and an emergency set is provided by the patient, as physician attendance may be delayed and as many physicians are reluctant to use the emergency set for still unknown reasons. Thus, self-injection holds the potential to greatly shorten the time to sufficient hydrocortisone coverage.

No ready-for-use hydrocortisone preparation is available so far; thus, preparation of hydrocortisone for injection takes time and patients may struggle to handle glass ampoules with powder and separate solvent and needles in case of emergency. Thus, development of an easy-to-handle prefilled syringe, preferably a pen, enabling the patient to quickly administer a sufficient amount of hydrocortisone as a single injection should be a future goal to effectively improve crisis management in adrenal insufficiency. To better standardize the pharmacokinetic studies, in our study, the injections were performed by the same investigators and not by the patients. However, in general practice, it is crucial that patients not only receive prescription of hydrocortisone ampoules but also receive structured training on how to perform self-injection.

In conclusion, our data indicate that s.c. emergency administration of hydrocortisone is feasible and efficacious with regard to target serum cortisol concentrations in incipient adrenal crisis. Future studies should clarify how both i.m. and s.c. injections perform under conditions of manifest circulatory insufficiency. Subcutaneous hydrocortisone may become an important new tool to improve the self-management of patients with adrenal insufficiency in the ambulatory setting.

\section{Supplementary data}

This is linked to the online version of the paper at http://dx.doi.org/10. 1530/EJE-12-1057.

\section{Declaration of interest}

The authors declare that there is no conflict of interest that could be perceived as prejudicing the impartiality of the research reported.

\section{Funding}

This work has been supported by the Else Kröner-Fresenius Stiftung (grant no. 2010_EKES.29 to S Hahner).

\section{Author contribution statement}

S Hahner was involved in design of study, preparation of study protocol and applications for authorities, conduct of the study, data analysis, and preparation of the manuscript. S Burger-Stritt was involved in preparation of applications for authorities, conduct of the study, laboratory measurements, and data analysis. B Allolio was involved in design of the study and preparation of the manuscript.

\section{Acknowledgements}

The authors thank Walter Rüger and the whole team of the Endocrine Outpatient Clinic, Department of Internal Medicine I, University Hospital Wuerzburg for their excellent cooperation.

\section{References}

1 White K \& Arlt W. Adrenal crisis in treated Addison's disease: a predictable but under-managed event. European Journal of Endocrinology 2010162 115-120. (doi:10.1530/EJE-09-0559)

2 Hahner S, Loeffler M, Bleicken B, Drechsler C, Milovanovic D, Fassnacht M, Ventz M, Quinkler M \& Allolio B. Epidemiology of adrenal crisis in chronic adrenal insufficiency: the need for new prevention strategies. European Journal of Endocrinology 2010162 597-602. (doi:10.1530/EJE-09-0884)

3 Kyle UG, Jolliet P, Genton L, Meier CA, Mensi N, Graf JD, Chevrolet JC \& Pichard C. Clinical evaluation of hormonal stress 
state in medical ICU patients: a prospective blinded observational study. Intensive Care Medicine 200531 1669-1675. (doi:10.1007/ s00134-005-2832-9)

4 Salluh JI, Bozza FA, Soares M, Verdeal JC, Castro-Faria-Neto HC, Lapa ESJR \& Bozza PT. Adrenal response in severe communityacquired pneumonia: impact on outcomes and disease severity. Chest 2008134 947-954. (doi:10.1378/chest.08-1382)

5 Desborough JP. The stress response to trauma and surgery. British Journal of Anaesthesia 200085 109-117. (doi:10.1093/bja/85.1.109)

6 Kudielka BM, Buske-Kirschbaum A, Hellhammer DH \& Kirschbaum C. HPA axis responses to laboratory psychosocial stress in healthy elderly adults, younger adults, and children: impact of age and gender. Psychoneuroendocrinology 200429 83-98. (doi:10.1016/S0306-4530(02)00146-4)

7 Hahner S, Spinnler C, Beuschlein F, Fassnacht M, Lang K, Quinkler M \& Allolio B. Adrenal crisis and general morbidity in chronic adrenal insufficiency prospectively assessed in 472 patients. Endocrine Abstracts 201126 OC1.5.

8 Bergthorsdottir R, Leonsson-Zachrisson M, Oden A \& Johannsson G. Premature mortality in patients with Addison's disease: a population-based study. Journal of Clinical Endocrinology and Metabolism 200691 4849-4853. (doi:10.1210/jc.2006-0076)

9 Bensing S, Brandt L, Tabaroj F, Sjoberg O, Nilsson B, Ekbom A, Blomqvist P \& Kampe O. Increased death risk and altered cancer incidence pattern in patients with isolated or combined autoimmune primary adrenocortical insufficiency. Clinical Endocrinology 200869 697-704. (doi:10.1111/j.1365-2265.2008. 03340.x)

10 Erichsen MM, Lovas K, Fougner KJ, Svartberg J, Hauge ER, Bollerslev J, Berg JP, Mella B \& Husebye ES. Normal overall mortality rate in Addison's disease, but young patients are at risk of premature death. European Journal of Endocrinology 2009160 233-237. (doi:10.1530/EJE-08-0550)
11 Wass JA \& Arlt W. How to avoid precipitating an acute adrenal crisis. BMJ 2012345 e6333. (doi:10.1136/bmj.e6333)

12 Sprung CL, Annane D, Keh D, Moreno R, Singer M, Freivogel K, Weiss YG, Benbenishty J, Kalenka A, Forst H et al. Hydrocortisone therapy for patients with septic shock. New England Journal of Medicine 2008358 111-124. (doi:10.1056/NEJMoa071366)

13 Ray DC, Macduff A, Drummond GB, Wilkinson E, Adams B \& Beckett GJ. Endocrine measurements in survivors and nonsurvivors from critical illness. Intensive Care Medicine $2002 \mathbf{2 8}$ 1301-1308. (doi:10.1007/s00134-002-1427-y)

14 Goodman S, Sprung CL, Ziegler D \& Weiss YG. Cortisol changes among patients with septic shock and the relationship to ICU and hospital stay. Intensive Care Medicine 200531 1362-1369. (doi:10.1007/s00134-005-2770-6)

15 Allolio B, Lang K \& Hahner S. Addisonian crisis in a young man with atypical anorexia nervosa. Nature Reviews. Endocrinology 20117 115-121. (doi:10.1038/nrendo.2010.211)

16 Oppert M, Reinicke A, Graf KJ, Barckow D, Frei U \& Eckardt KU. Plasma cortisol levels before and during "low-dose" hydrocortisone therapy and their relationship to hemodynamic improvement in patients with septic shock. Intensive Care Medicine 2000 26 1747-1755. (doi:10.1007/s001340000685)

17 Voortman G, Mannaerts BM \& Huisman JA. A dose proportionality study of subcutaneously and intramuscularly administered recombinant human follicle-stimulating hormone (Follistim*/ Puregon) in healthy female volunteers. Fertility and Sterility 200073 1187-1193. (doi:10.1016/S0015-0282(00)00542-2)

Received 5 December 2012

Revised version received 15 April 2013

Accepted 14 May 2013 